\title{
EFFECTIVE HYDRAULIC CONDUCTIVITY FOR A SOIL OF VARIABLE PORE SIZE WITH DEPTH
}

\author{
C. C. Nnaji*

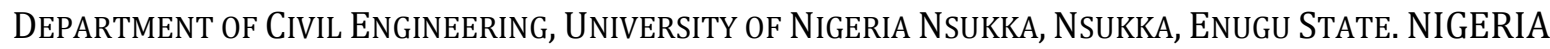 \\ E-mail address: chidozie.nnaji@unn.edu.ng
}

\begin{abstract}
Two models were derived for the estimation of effective hydraulic conductivity $\left(K_{e}\right)$ of a soil layer based on exponential and inverse square variation of hydraulic conductivity with soil depth. Darcy's law was applied to a vertical soil stratum subdivided into a finite number of layers. The relationship between $K_{e}$ and layer thickness is of quadratic form with $R^{2} \approx 1$.As the layer thickness increases, the values of $K_{e}$ for the exponential model increases drastically, exceeding the $K_{e}$ estimate of the power model. The percentage difference between the two models assumes an asymptotic form to the $y$-axis at a percentage difference of $5 \%$, as the size of layer approaches zero. power model gives lower estimates of $K_{e}$ than the exponential model within soil depth range of $1.5 m \leq D \leq 3.2 m$ for $n=2$; $0.8 \mathrm{~m} \leq D \leq 1.9 \mathrm{~m}$ for $n=50$; and $0.7 \mathrm{~m} \leq D \leq 1.9 \mathrm{~m}$ for $n=100$ and above. For very shallow soil stratum $(D \leq 2)$, the exponential model gives better and more realistic estimates of $K_{e}$ than the power model; while for medium to deep soil stratum $(D \geq 2)$, the power model gives better estimates of $K_{e}$
\end{abstract}

Keywords: hydraulic conductivity, soil, infiltration, permeability, water

\section{INTRODUCTION}

Accurate determination of hydraulic conductivity is very crucial for infiltration and runoff estimation. Factors which affect water infiltration in the soil include hydraulic conductivity, wetting front and soil sorptivity [1], ponding depth [2], depth to water table [3] and impact of rain drops [4]. Amongst these factors, the importance of hydraulic conductivity cannot be overemphasized. Hydraulic conductivity is a very important soil parameter used by the Green-Ampt model for estimation of infiltration and runoff. It is basically an intrinsic property of the soil and [5] summarized the hydraulic conductivities of various textural classes of soil. Selker [6] observed that though soil hydraulic conductivity decreases with soil depth from the surface, yet many infiltration models ignore this fact. Beven [7] was the first to apply this fact to a small catchment by expressing both hydraulic conductivity and soil moisture content as a function of soil depth as: $K_{S}(Z)=K *(D-Z)^{n}$ and $\theta_{S}(Z)=\theta *$ $(D-Z)^{m}$. Here, $K_{S}$ is the saturated hydraulic conductivity, $\theta_{\mathrm{s}}$ is the saturated moisture content, $Z$ is increasing depth of soil for a soil of total soil depth $(D)$ while $K * \theta * n$, and $m$ are site specific soil parameters to be fitted to soil data. The paper [8] recognized the fact that heterogeneity of hydraulic conductivity is a significant driver of net basin infiltration.

One would ordinarily assume that decrease in soil permeability with depth is as a result of decreasing porosity resulting from greater packing density of soil particles. However, the experimental results of [9] refute this supposition. Selkar [6] also found that hydraulic conductivity is affected by characteristic pore size rather than porosity. He observed that as pore size decreases, hydraulic conductivity decreases with square of pore size. Askari, et al [10] noted that hydraulic conductivity is influenced by the presence of organic matter and montmorillonitic mineral materials. While there exists a gradual variation of hydraulic conductivity through a homogeneous soil profile, [11] noted that soil hydraulic properties can vary in a nested fashion as a result of surface disturbances due to tillage, pore size distribution due to structural cracks and root development and decay, textural layering and geology. This is usually the case in agricultural soils. Variability in soil hydraulic conductivity is more pronounced and sporadic in agricultural soils as a result of alterations of soil 
structure and properties during agricultural activities. It was in recognition of this that [4] employed empirical approach to estimate effective hydraulic conductivity under meadows conditions. Leconte and Briseette [12] also observed that the impact of rain drops on bare soil surfaces give rise to stable crust layer, having a saturated hydraulic conductivity significantly lower than soil below it.

Having adequately established that hydraulic conductivity of soils is as variable as many other soil properties, the objective of this paper is to obtain simple and easy to apply expressions for effective hydraulic conductivity in a soil of uniform profile. The traditional infiltration models use a constant hydraulic conductivity to evaluate infiltration and this possibly accounts for disparity between calculated and field infiltration results.

\section{METHODOLOGY}

Figure 1 below shows the vertical profile of a homogeneous soil stratum of depth $D$ whose hydraulic conductivity gradually decreases in the vertical direction. This stratum has been subdivided into $n$ minute layers of thickness $Z$, each of which is assumed to have a constant hydraulic conductivity. The hydraulic conductivity of each minute layer is represented by the average of the hydraulic conductivities of the immediate upper and lower borders. First we derive the expression for effective $K$ for $n$ finite layers of clearly distinct $K$.

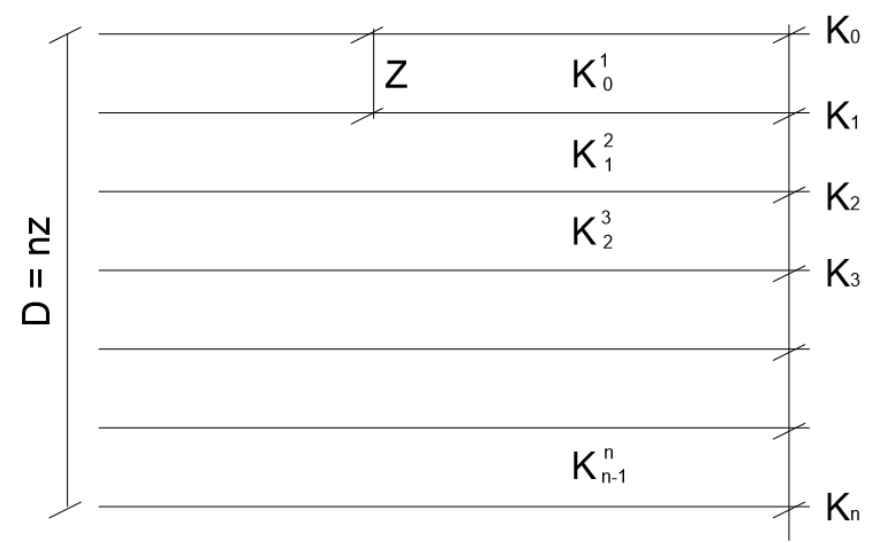

Figure 1: Soil Stratum Subdivided Into Layers

According to Darcy's law,

$$
Q=K A \frac{d h}{d L}
$$

Therefore, $d h=\frac{Q}{K A} d L=\frac{Q}{K A} Z$. The total headloss through the layers is given by $d h=d h_{1}+d h_{2}+d h_{3}$ $+\ldots . . d h_{n}$. Hence, for equal layers of soil:

$$
\begin{gathered}
\frac{Q(n Z)}{K_{e} A}=\frac{Q Z}{K_{1} A}+\frac{Q Z}{K_{2} A}+\cdots+\frac{Q Z}{K_{n} A} \\
\therefore \frac{n Z}{K_{e} A}=\frac{Z}{K_{1}}+\frac{Z}{K_{2}}+\cdots+\frac{Z}{K_{n}} \\
\therefore \frac{n}{K_{e}}=\sum_{i-1}^{n} \frac{1}{K_{i}}
\end{gathered}
$$

Since a representative value of $K$ is required for each layer, it can safely be assumed that for the infinitesimal layer between any two boundaries, say 0 and 1 ,

$$
\begin{gathered}
K_{0}^{1}=\frac{K_{0}+K_{1}}{2} ; . K_{1}^{2}=\frac{K_{1}+K_{2}}{2} ; . K_{2}^{3}=\frac{K_{2}+K_{3}}{2} ; . K_{i-1}^{i} \\
=\frac{K_{i-1}+K_{i}}{2}
\end{gathered}
$$

Substituting Equation (5) into Equation (4), we have:

$$
\frac{n}{K_{e}}=\sum_{i=1}^{n} \frac{2}{K_{i-1}+K_{i}}
$$

An expression is needed for the variation of $K$ with depth. Two cases were considered: (i) a power law $K(D)=K_{0}(\beta D)^{-2}$ and (ii) an exponential function $K(D)=K_{0} \exp (-2 \beta D)$ as given by [6]

\subsection{Power Law}

Beginning from the first layer and using $n$ layers such that $D=n Z, K_{1}=K_{0} \beta Z^{-2}, K_{2}=K_{0}(\beta 2 Z)^{-2}$,

$K_{3}=K_{0}(\beta 3 Z)^{-2}$

Hence

$$
K_{n}=K_{0}(\beta n Z)^{-2}
$$

Also

$$
\begin{aligned}
& K_{i-1}^{i}=K_{0} \beta^{-2} Z^{-2}(i-1)^{-2}+K_{0} \beta^{-2}(Z i)^{-2} \\
& K_{i-1}^{i}=K_{0} \beta^{-2} Z^{-2}\left(\frac{1}{i^{2}}+\frac{1}{(1-i)^{2}}\right)
\end{aligned}
$$

Substituting Equation (9) in Equation (6):

$$
\begin{aligned}
\frac{n}{K_{e}} & =\sum_{i=1}^{n} \frac{2}{K_{0} \beta^{-2} Z^{-2}\left(\frac{1}{i^{2}}+\frac{1}{(1-i)^{2}}\right)} \\
\frac{n}{K_{e}} & =\frac{2 \beta^{2} Z^{2}}{K_{0}} \sum_{i=1}^{n} \frac{1}{\left(\frac{1}{i^{2}}+\frac{1}{(1-i)^{2}}\right)}
\end{aligned}
$$

Recalling that $D=n Z$ :

$$
\frac{1}{K_{e}}=\frac{2 \beta^{2} Z^{2}}{n^{2} K_{0}} \sum_{i=1}^{n} \frac{1}{\left(\frac{1}{i^{2}}+\frac{1}{(1-i)^{2}}\right)}
$$

The term $\frac{1}{i^{2}}+\frac{1}{\left(\frac{1}{(1-i)^{2}}\right)}$ can be simplified as follows:

$$
\frac{1}{i^{2}}+\frac{1}{(1-i)^{2}}=\frac{2 i^{2}-2 i+1}{i^{2}\left(i^{2}-2 i+1\right)}
$$

Hence, Equation (12) becomes: 


$$
\begin{gathered}
\frac{1}{K_{e}}=\frac{2 \beta^{2} Z^{2}}{n^{2} K_{0}} \sum_{i=1}^{n} \frac{i^{2}\left(i^{2}-2 i+1\right)}{2 i^{2}-2 i+1} \\
\sum_{i=1}^{n} \frac{i^{2}\left(i^{2}-2 i+1\right)}{2 i^{2}-2 i+1} \Rightarrow \sum_{i-1}^{n} \frac{i^{2}\left(i^{2}-2 i+1\right)}{2 i^{2}-2 i} \\
=\frac{1}{2} \sum_{i=1}^{n}\left(i^{2}-i\right)
\end{gathered}
$$

As $n$ becomes very large, the solution converges,

The expression $\frac{1}{2} \sum_{i-1}^{n}\left(i^{2}-i\right)$ can be split into two as follows:

$$
\frac{1}{2} \sum_{i=1}^{n}\left(i^{2}-i\right) \frac{1}{2}=\left[\sum_{i=1}^{n} i^{2}+\sum_{i=1}^{n} i\right]
$$

The two sum of series can further be simplified as follows:

$$
\begin{aligned}
& \sum_{i=1}^{n} i=\frac{n(n+1)}{2} \\
& \sum_{i=1}^{n} i^{2}=\frac{n(n+1)(2 n+1)}{6}
\end{aligned}
$$

Hence Equation (14) becomes:

$$
\begin{gathered}
\frac{1}{K_{e}}=\frac{\beta^{2} D^{2}}{n^{3} K_{0}}\left[\frac{n(n+1)}{2}+\frac{n(n+1)(2 n+1)}{6}\right] \\
K_{e}=\frac{3 K_{0}}{\beta^{2} D^{2}}\left[\frac{n^{2}}{n^{2}-1}\right]
\end{gathered}
$$

Let the layer thickness $\frac{D}{n}=\alpha$

$$
K_{e}=\frac{3 K_{0}}{\beta^{2} \alpha^{2}}\left[\frac{1}{n^{2}-1}\right]
$$

\subsection{Exponential Function}

Following the steps already introduced for the power function above, the exponential variation of hydraulic conductivity $(K)$ through the soil profile for the $n^{\text {th }}$ layer can be expressed as:

$$
\begin{gathered}
K_{n}=K_{0} \exp (-2 \beta(n Z)) \\
K_{i-1}^{i}=K_{0}[\exp (-2 \beta Z(i-1))+\exp (-2 \beta Z i)]
\end{gathered}
$$

Substituting Equation (24) in Equation (4), we obtain:

$$
\begin{gathered}
\frac{n}{K_{e}}=\sum_{i=1}^{n} \frac{2}{\exp (-2 \beta Z i)[1+\exp (-2 \beta Z)]} \\
K_{e}=\frac{n K_{0}[1+\exp (-2 \beta D / n)]}{2 \sum_{i=1}^{n} \exp \left(\frac{2 \beta D i}{n}\right)}
\end{gathered}
$$

The numerator can further be simplified as follows:

$$
\begin{aligned}
\sum_{i=1}^{n} \exp \left(\frac{2 \beta D i}{n}\right) & =\exp \left(\frac{2 \beta D}{n}\right)+\exp \left(\frac{4 \beta D}{n}\right) \\
& +\exp \left(\frac{6 \beta D}{n}\right)+\exp \left(\frac{8 \beta D}{n}\right)+\cdots \\
& +\exp (2 \beta D)
\end{aligned}
$$

Equation (26) can easily be recognized as the sum of a geometric progression with the following properties.

$$
\alpha=r=\exp \left(\frac{2 \beta D}{n}\right)
$$

Hence

$$
\sum_{i=1}^{n} \exp \left(\frac{2 \beta D i}{n}\right)=\exp \left(\frac{2 \beta D}{n}\right)\left[\frac{1-\exp (2 \beta D)}{1-\exp \left(\frac{2 \beta D i}{n}\right)}\right]
$$

Substituting Equation (27) in Equation (25) yields

$$
\begin{gathered}
K_{e}=\frac{K_{0}[1-\exp ]}{2 \exp \left(\frac{2 \beta D}{n}\right)\left[1-\exp \left(\frac{2 \beta D}{n}\right)\right]} \\
K_{e}=\frac{n K_{0}}{2[1-\exp (2 \beta D)]}\left[\exp \left(-\frac{2 \beta D}{n}\right)\right] \\
-\exp \left(\frac{2 \beta D}{n}\right)
\end{gathered}
$$

In terms of layer thickness, we have

$$
K_{e} \frac{n K_{0}}{2[1-\exp (2 \beta D)]}[\exp (-2 \beta \alpha)-\exp (2 \beta \alpha)]
$$

Equations (21) and (31) are the expressions for effective hydraulic conductivity for a uniformly varying saturated soil, assuming power and exponential functions respectively.

\section{RESULTS AND DISCUSSION}

The models were illustrated using the experimental data obtained by [9]. Of course this was for a specific soil. The use of Equations (21) and (32) will require knowing the values of the soil parameters $K_{o}$ and $\beta$. For the power function (Equation 30), these parameters can be obtained by rearranging the expression $K(D)=$ $K_{0}(\beta D)^{-2}$ as follows:

$$
\operatorname{Ln}(K)=\operatorname{Ln}\left(\frac{1}{Z^{2}}\right)+\operatorname{Ln}\left(K_{0} \beta^{-2}\right)
$$

The plot of $\operatorname{Ln}(K)$ versus $\operatorname{Ln}\left(\frac{1}{Z^{2}}\right)$ is a fairly straight line with intercept $\operatorname{Ln}\left(K_{0} \beta^{-2}\right)$ as shown in Figure 2. Hence, the value of $K_{0} \beta^{-2}$ can be determined.

From the plot shown in Figure 2, the $\operatorname{Ln}\left(K_{0} \beta^{-2}\right)=$ 0.982 so that $K_{0} \beta^{-2}=2.67 \mathrm{~m}^{3} / \mathrm{hr}$. For the exponential model, the soil parameters were determined by rearranging the expression $K(D)=K_{0} \exp (-2 \beta D)$ as follows:

$$
\operatorname{Ln}(K)=\operatorname{Ln} K_{0}-2 \beta Z
$$




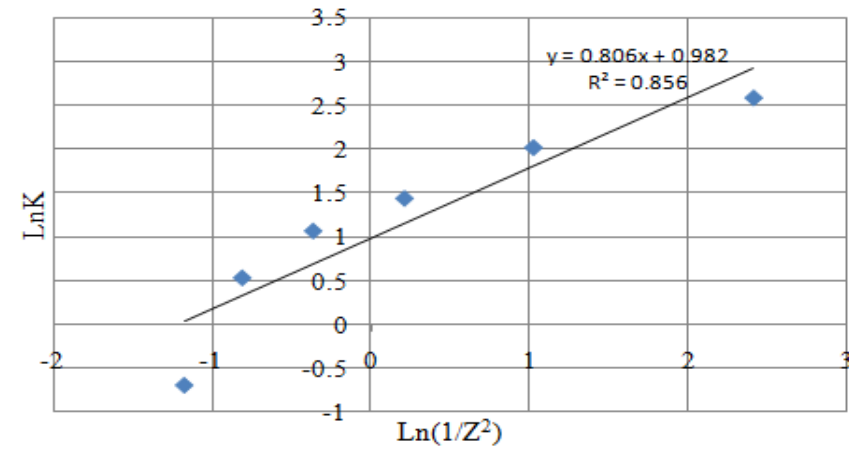

Figure 2: Plots for Determination of Soil Parameters for the Power Model

From the plot of $L n K$ versus $Z$ shown in Figure 3 below, the values of $K_{0}$ and $\beta$ can be respectively determined as $26.42 \mathrm{~m} / \mathrm{hr}$ and $1.009 / \mathrm{m}$.

In order to ascertain the effect of layer size on the values of $K_{e}$, plots of $K_{e}$ versus layer thickness were produced as shown in Figure 4. As the layer thickness becomes infinitesimal $(\alpha \rightarrow 0)$, the plot of $K_{e}$ versus layer thickness becomes asymptotic to the layer thickness axis for the two models. However, as the layer thickness increases, the values of $K_{e}$ for the exponential model increases drastically, exceeding the $K_{e}$ estimate of the power model. Figure 4 also shows that the relationship between $K_{e}$ and layer thickness is of quadratic form with $R^{2} \approx 1$. As is to be reasonably expected, better estimates of $K_{e}$ can be obtained by subdividing the soil stratum into infinitesimal layers. To further understand the effect of soil stratum subdivision on accurate estimation of $K_{e}$, a plot of $K_{e}$ versus number of layers (n) was produced a shown in Figure 5. Figure 5 shows that the power model consistently gives lower values of $K_{e}$ than the exponential model. However, the rate of convergence is identical in both cases. Both models converged when the soil was subdivided into twenty (20) layers, with further subdivision (up to $\mathrm{n}=100$ ) resulting in no further improvement in $K_{e}$ values. Also as n approaches 1 , the $K_{e}$ estimate of the power model tends to infinity. This reveals a structural deficiency in the underlying assumption that hydraulic conductivity varies with the inverse square of depth - the relationship is not that simple.

Figure 6 is a plot of number of layers and size of layers versus percentage difference between the $K_{e}$ values of the two models. As the size of layer increases, the percentage difference increases, approaching $25 \%$ at a layer size of $1 \mathrm{~m}$ and $\mathrm{n}=2$. However, percentage difference between the two models assumes an asymptotic form to the $y$-axis at a percentage difference

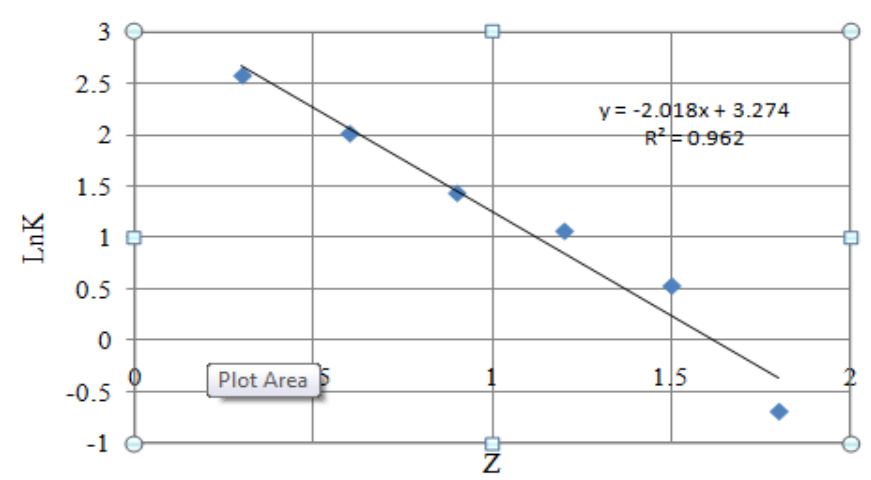

Figure 3: Plots for Determination of Soil Parameters for the Exponential Model

of $5 \%$, as the size of layer approaches zero. The percentage difference drastically reduces to approximately $5 \%$ at a layer size of $0.36 \mathrm{~m}$. This shows that the models become very close as layer size becomes infinitesimal, but can never completely agree. Likewise, as the number of layers increases, the percentage difference approaches 5\% asymptotically; and as the number of layers increases, the percentage difference increases drastically. A close look at Equation (20) shows that it converges to a definite value as $n \rightarrow \infty$. This eliminates the need for subdividing the soil stratum into layers and shorten the computation time. Hence as $n \rightarrow \infty$,

$$
K_{e}=\frac{3 K_{0}}{\beta^{2} D^{2}}
$$

For the soil parameters used in this research, the value of $K_{e}$ obtained by using Equation (33) is $2.477 \mathrm{~m} / \mathrm{hr}$. If the $K_{e}$ were to be determined by subdividing the soil stratum, it would require hundred layers for the power law model to yield this value. The exponential model gave a value of $2.608 \mathrm{~m} / \mathrm{hr}$ using hundred layers. Figure 5 shows that both the power and exponential models tend towards the $K_{e}$ estimate of Equation (33). Hence it can be surmised that Equation (33), though simple in nature, gives a reliable estimate of $K_{e}$ Figure 7 shows the percentage difference between the $K_{e}$ values of the two models using different number of layers and layer sizes, and the $K_{e}$ estimate of Equation (33). Figure 7a shows that the percentage difference for the power model quickly approaches zero even at an $n$ value of 30 and is proportional to the inverse of the square of the number of layers. Doubling the number of layers, reduces the percentage difference by a quarter (25\%). On the other hand, doubling the number of layers for exponential model reduces the percentage difference by about $30 \%$; but the percentage difference does not exceed $5 \%$ even at an $n$ value of 1000 . Generally, the exponential model gives higher values of percentage 
difference than the power model, for the same number of layers.

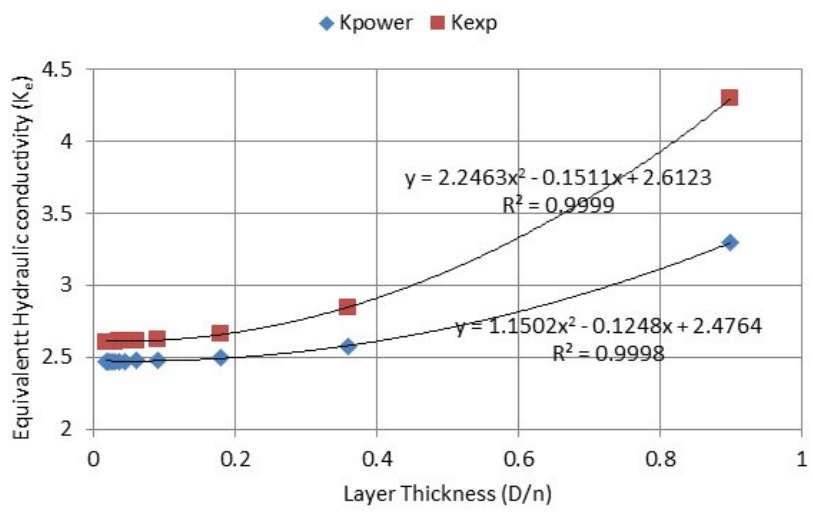

Figure 4: Effective Hydraulic Conductivity versus Layer Thickness.

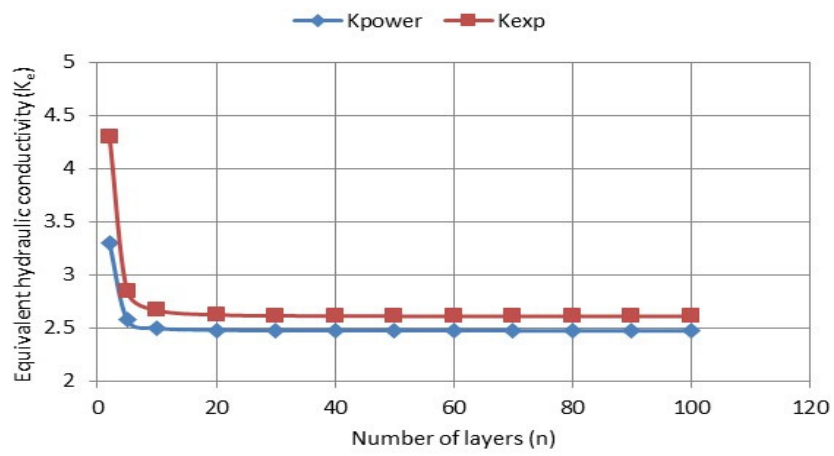

Figure 5: Effective Hydraulic Conductivity versus Number of Layers

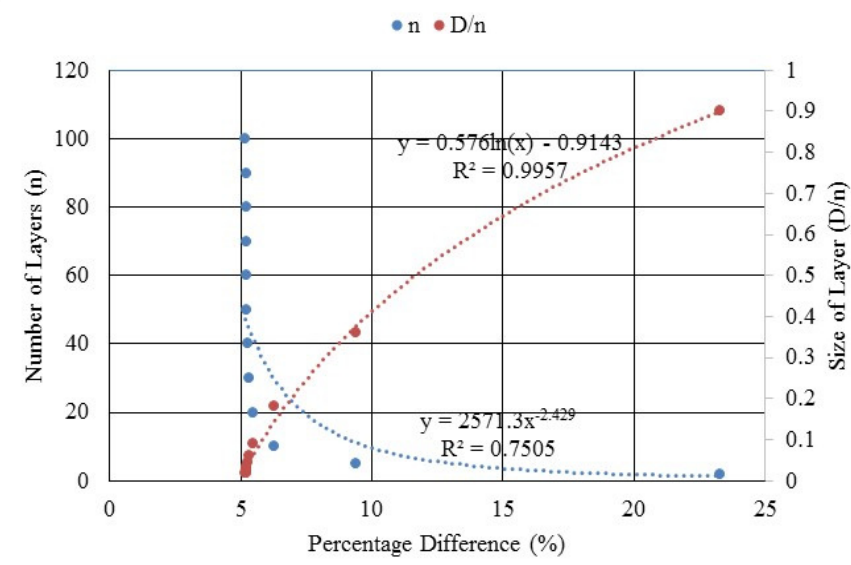

Figure 6: Percentage Difference between the Power and Exponential Models Estimate of $K_{e}$

Further investigations were made in order to understand the behavior of the two models with respect to the depth of soil stratum and the number of subdivisions. Generally, the $K_{e}$ estimates of the two models become increasingly smaller as the soil depth increases. Figure 8 clearly shows that the power model gives lower estimates of $K_{e}$ than the exponential model within soil depth range of $1.5 \mathrm{~m} \leq D \leq 3.2 \mathrm{~m}$ for $\mathrm{n}=2$; $0.8 \mathrm{~m} \leq D \leq 1.9 \mathrm{~m}$ for $\mathrm{n}=50$; and $0.7 \mathrm{~m} \leq D \leq 1.9 \mathrm{~m}$ for $\mathrm{n}=100$ and above. For all other ranges of depth, the exponential model gives lower $K_{e}$ estimates. Besides for shallow soil stratum, the power model cannot be relied upon for good estimates of $K_{e}$ because the curve becomes asymptotic to the $K_{e}$ axis as soil depth reduces. This is especially the case when very few subdivisions are used. Moreover, as soil depth increases, the $K_{e}$ estimates of the exponential model approach zero. This too is unreliable. Hence it can be clearly seen that for very shallow soil stratum $(D \leq 2)$, the exponential model gives better and more realistic estimates of $K_{e}$ than the power model; while for medium to deep soil stratum $(D \geq 2)$, the power model gives better estimates of $K_{e}$.

\section{CONCLUSIONS}

Two simple models for estimating hydraulic conductivity of uniform soils have been derived. The result of this research has shown that hydraulic conductivity does not exactly follow the inverse square law for shallow soils. Moreover, the two models are complementary to each other for varying ranges of soil depth. Besides, an infinite number of soil layers is not necessarily required for the solution to converge. Overall, the power model is more advantageous than the exponential model in that it converges to a finite value for an infinite number of subdivisions. This gave rise to Equation (33) which does not require layer subdivision and yet yields good results.

\section{REFERENCES}

[1] Regalado, C. M., Ritter, A., Alvarez-Benedi, J. and Munōz-Carpena, R. "Simplified method to estimate the Green-Ampt wetting front suction and soil sorptivity with the Philip-Dune falling head permeameter", Vadoze Zone Journal, Vol. 4,2005, pp291 - 299.

[2] Warrick, A. W., Derihun, D. Sanchez, C. A. and Furman, A. "Infiltration under variable ponding depths of water", Journal of Irrigation and Drainage Engineering Vol. 131, Number 4, 2005, pp 358 - 363.

[3] Risse, L. M., Nearing, M. A. and Savabi, M. R. "Determining the Green-Ampt effective hydraulic conductivity from rainfall-runoff data for the WEPP mode", Transactions of the ASAE, Vol. 37, Number 2, 1994, 411 - 418.

[4] Zhang, X. C., Nearing, M. A. and Risse, L. M. "Estimation of Green-Ampt conductivity parameters: Part II Perennial crops, Transactions of the ASAE, Vol. 38, Number 4, 1995, pp1079-1087.

[5] Rawls, W. J., Brakensiek, D. L. and Miller, N. "GreenAmpt infiltration parameters from soil data", Journal of Hydraulic Engineering, Vol. 109, Number 1, 1983, pp62 - 70 . 

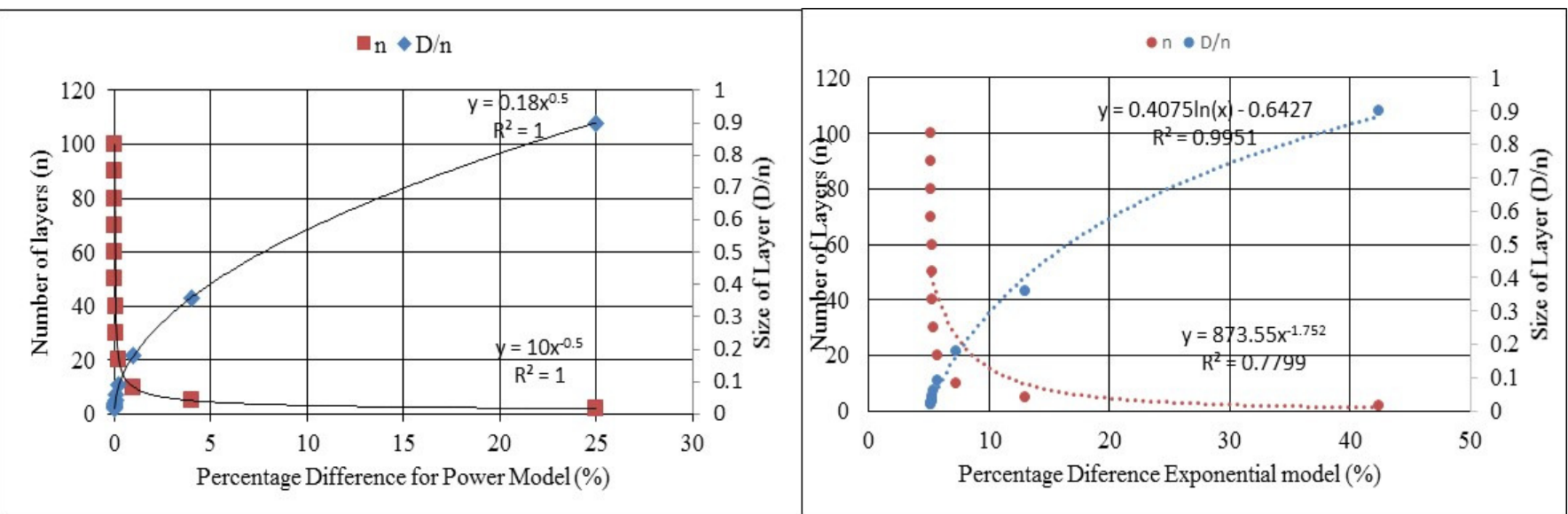

Figure 7: Comparison of the Models Estimates of $K_{e}$ with that of Equation (33)
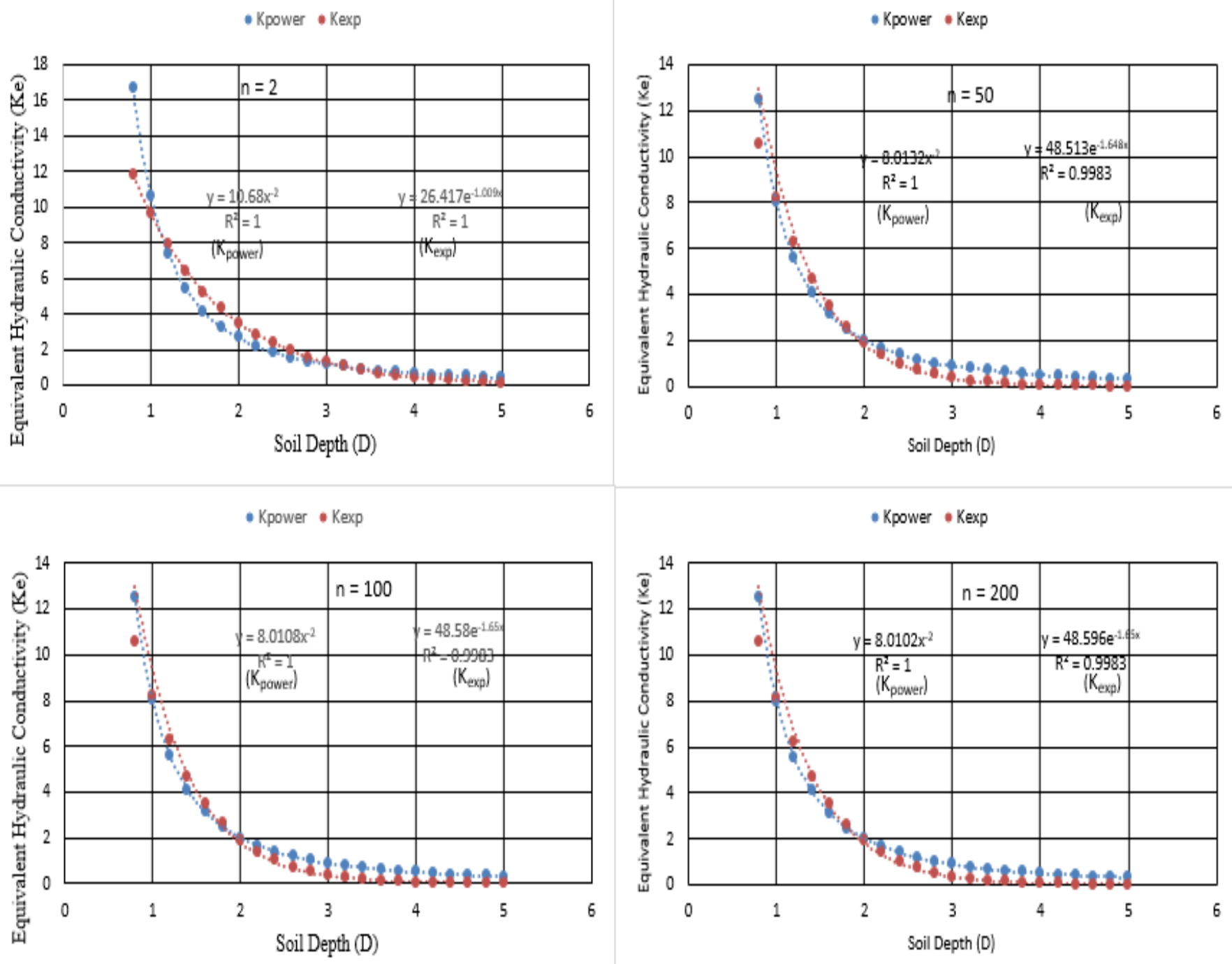

Figure 8: Plots of $K_{e}$ versus Soil Depth Using Different Number of Soil Layers.

[6] Selker, S. J. “Green and Ampt infiltration into soils of variable pore size with depth", Water Resources Research, Vol. 35, Number 5,1999, pp1685 - 1688.
[7] Beven, K. "Infiltration into A Class of Vertically NonUniform Soils", Hydrological Sciences Journal, Vol. 29,1984 , pp $425-434$.

[8] Craig, J. R., Liu, G. and Soulis, E. D. “Runoff-infiltration partitioning using an upscaled Green-Ampt 
solution”, Hydrological Processes, doi:10.1002/hyp.7601, 2010.

[9] Childs, E. C. and Bybordi, M. (1969), “The vertical movement of water in stratified porous material, I, Infiltration", Water Resources Research, Vol. 5, 1969, pp446 - 459.

[10] Askari, M. Tanaka, T., Setiwan, B. and Saptomo, S. K. "Infiltration characteristics of tropical soil based on water retention data", Journal of Japan Society of Hydrology and Water Resources, Vol. 21, Number 3,2008 , pp215 - 227.
[11] Mohanty, B. P. and Zhu, J. "Effective hydraulic parameters in horizontally and vertically heterogonous soils for steady-state landatmospheric interaction", Journal of Hydrometeorology, Vol. 8, 2007, pp715 - 729.

[12] Leconte, R. and Brisseette, F. “Soil moisture profile mdel for two-layered soil based on sharp wetting front approach", Journal of Hydrologic Engineering, Vol. 6, Number 2, 2001, pp141 - 149. 